\title{
Towards a Metallurgy of Neutron Star Crusts
}

\author{
D. Kobyakov ${ }^{1,2,3}$ and C. J. Pethick ${ }^{3,4}$ \\ ${ }^{1}$ Department of Physics, Umeå University, SE-901 87 Umeå, Sweden \\ ${ }^{2}$ Radiophysics Department, Nizhny Novgorod State University, Gagarin Avenue 23, 603950 Nizhny Novgorod, Russia \\ ${ }^{3}$ The Niels Bohr International Academy, The Niels Bohr Institute, University of Copenhagen, \\ Blegdamsvej 17, DK-2100 Copenhagen $\emptyset$, Denmark \\ ${ }^{4}$ NORDITA, KTH Royal Institute of Technology and Stockholm University, Roslagstullsbacken 23, SE-106 91 Stockholm, Sweden
}

(Received 16 August 2013; published 21 March 2014)

\begin{abstract}
In the standard picture of the crust of a neutron star, matter there is simple: a body-centered-cubic lattice of nuclei immersed in an essentially uniform electron gas. We show that, at densities above that for neutron drip ( $\sim 4 \times 10^{11} \mathrm{~g} \mathrm{~cm}^{-3}$ or roughly one-thousandth of nuclear matter density), the interstitial neutrons give rise to an attractive interaction between nuclei that renders the lattice unstable. We argue that the likely equilibrium structure is similar to that in displacive ferroelectric materials such as $\mathrm{BaTiO}_{3}$. As a consequence, the properties of matter in the inner crust are expected to be much richer than previously appreciated, and we mention possible consequences for observable neutron star properties.
\end{abstract}

PACS numbers: 21.65.-f, 26.60.Gj, 67.10.Jn, 97.60.Jd

Many technologically important properties of terrestrial metals are governed by the fact that these materials exhibit a variety of crystal structures. Pure metals have many different phases [1]. For alloys, even more possibilities exist, and these have far-reaching implications: e.g., the strength of steels is determined to a high degree by the existence of different crystal structures. Here we consider matter in the outer parts of a neutron star (its crust), which is important for interpreting observations of neutron stars even though it comprises only a small fraction of the total mass of the star. In the traditional view, this matter is simple, because correlations between electrons, which are crucial for terrestrial matter, play little role. However, at densities above one-thousandth of nuclear density, matter consists of a crystal lattice of atomic nuclei permeated by neutrons [2]. The neutrons behave like a second component in a binary alloy, and we argue that, as a consequence, the properties of matter are more similar to those of terrestrial solids than has been previously appreciated. Specifically, the neutrons give rise to an attractive interaction between nuclei which makes the lattice unstable to clumping of nuclei in a manner similar to the formation of inhomogeneous regions in metallic alloys (spinodal decomposition) [3]. While the attraction is insufficient to make matter unstable to long-wavelength distortions, it can destabilize matter at finite wavelengths where the effective interaction between nuclei due to their electrical charges is reduced. We describe a number of possible consequences for observable properties of neutron stars.

To set the scene, we consider the condition for thermodynamic stability of the system of nuclei immersed in a sea of neutrons, together with a background of electrons whose average density is the same as that of the protons to ensure electrical neutrality. The system may thus be regarded as having two components: the neutrons (both those in nuclei and the interstitial ones) and the charged particles. For most of the life of a neutron star, the temperature is so low that thermal effects may be neglected. In that case, the condition for stability is that the second-order change in the energy density be positive for neutron and proton densities slightly different from the initial ones (which are determined by the condition that matter is in equilibrium to weak interaction processes), i.e.,

$$
\delta^{2} E=\frac{1}{2} \sum_{i, j} \delta n_{i} \delta n_{j} E_{i j}>0
$$

where $E_{i j}=\partial^{2} E / \partial n_{i} \partial n_{j}$ [4]. The species labels $i$ and $j$ here refer to neutrons $(n)$ and protons $(p)$, and the $n_{i}$ are particle number densities. For electrically neutral matter, the electron density $n_{e}$ is equal to the proton density and is therefore not an independent variable. The quantity $E\left(n_{n}, n_{p}\right)$ is the energy of the system, including electrons, per unit volume. Sufficient conditions for stability are that one of the diagonal elements of $E_{i j}$ be positive and that the determinant of the matrix, $E_{p p} E_{n n}-E_{n p}^{2}$, be positive. Since $E_{n n}>0$, the latter condition may be rewritten as

$$
E_{p p}-\frac{E_{n p}^{2}}{E_{n n}}=\left.\frac{\partial \mu_{p}}{\partial n_{p}}\right|_{\mu_{n}}>0
$$

where the $\mu_{i}$ are chemical potentials. Equation (2) has the physical interpretation that the effective proton-proton interaction be positive [5]: the first term is usually referred to as the direct interaction, because it represents the interaction when the neutron density is held fixed, while the second term, the so-called induced interaction, is the contribution to the interaction due to the change in the 
neutron density. The second term, which is attractive, is similar to the phonon-induced interaction between electrons that is responsible for conventional superconductivity in terrestrial metals, except that in a neutron star the exchanged phonon is a neutron density fluctuation [6].

Calculation of the derivatives $E_{i j}$ is a challenge, because the system has two phases: the nuclei and the neutrons between nuclei. Consequently, the derivatives are not simply related to those of bulk nuclear matter. In order to obtain thermodynamically consistent results, we have recently evaluated them from Lattimer and Swesty's microscopic calculations of the properties of dense matter $[7,8]$ and thereby take into account the fact that, when neutrons are added to the system, they are distributed between the two phases. Our results show that matter is stable for bulk disturbances, since the magnitude of the induced interaction is typically no more than $0.2-0.3$ times the direct interaction [9]. The stability is due to the fact that, when the proton density is increased, the electron density must also increase and the effective proton-proton interaction is dominated by the energy required to compress the electrons.

The situation is different at finite wavelengths, or nonzero wave number $k$. The direct proton-proton interaction has two contributions. The first of these is due to the Coulomb interaction, and the dominant term for small $k$ comes from density variations at the same wave number. This is screened by the electrons and is given by [10]

$$
V(k)=\frac{V_{0}(k)}{1+V_{0}(k) \chi_{e}(k)},
$$

where $V_{0}(k)=4 \pi e^{2} / k^{2}$ is the bare Coulomb interaction, $e$ being the elementary charge, and $\chi_{e}(k)$ is the electron density-density response function. For small $k, \chi_{e}(k)$ tends to $\partial n_{e} / \partial \mu_{e} \equiv 1 / E_{e e}$, and therefore $V(k)$ tends to $\partial \mu_{e} / \partial n_{e}$. Inserting standard results for a relativistic gas, one finds that, for $k$ much less than the electron Fermi wave number $k_{e}$, the effective proton-proton interaction is given by (see Chap. 2 in [2])

$$
V(k)=\frac{V(0)}{1+k^{2} / k_{\mathrm{TF}}^{2}},
$$

where the Thomas-Fermi screening wave number is given by $k_{\mathrm{TF}}^{2}=(4 \alpha / \pi) k_{e}^{2}, \alpha=e^{2} / \hbar c$ being the fine structure constant. The wave number $k_{\mathrm{TF}}$ is smaller than the wave number at the boundary of the first Brillouin zone along a cubic axis: for a bcc structure, $k_{\mathrm{BZ}}=2 \pi / a$ with $a$ being the spacing of nuclei at the corners of a cubic cell, and $k_{\mathrm{TF}} / k_{\mathrm{BZ}}=\left(6^{1 / 3} / \pi^{5 / 6}\right) \alpha^{1 / 2} Z^{1 / 3} \approx 0.2$. Consequently, the $k$ dependence of the interaction is very important on the scale of the first Brillouin zone. There is also a Coulombic contribution $E_{p p}$ from umklapp processes, but, as calculations of phonon frequencies for a Coulomb lattice demonstrate [11], this varies on a wave number scale $\sim k_{\mathrm{BZ}}$ so it is a reasonable first approximation to regard the umklapp contribution as constant.

The second contribution to $E_{p p}$ is due to the strong interaction between nucleons, and this too we would expect to vary with $k$ with a characteristic scale $k_{\mathrm{BZ}}$, so we shall also neglect its dependence on $k$. We therefore take $E_{p p}$ at a nonzero wave number to be the screened Coulomb interaction (4) plus a constant term whose magnitude is chosen to give the correct limit for $k \rightarrow 0$ :

$$
\begin{aligned}
E_{p p}(k) & =\frac{\partial \mu_{e}}{\partial n_{e}} \frac{1}{1+k^{2} / k_{\mathrm{TF}}^{2}}+\left(E_{p p}-\frac{\partial \mu_{e}}{\partial n_{e}}\right) \\
& =E_{p p}-\frac{\partial \mu_{e}}{\partial n_{e}} \frac{k^{2}}{k_{\mathrm{TF}}^{2}+k^{2}} .
\end{aligned}
$$

This equation expresses the fact that at finite wavelengths it is less costly energetically to create proton density modulations, because electrons do not follow the protons if the wave number is comparable to or greater than $k_{\mathrm{TF}}$.

To investigate stability at nonzero $k$, we first assume that the instability condition is a direct generalization of Eq. (2) to this case. We expect the $k$ dependence of $E_{n n}$ and $E_{n p}$ to be on wave number scales of the order of $k_{\mathrm{BZ}}$, and therefore we replace these parameters by their $k=0$ values. The smallest unstable wave number $k_{c}$ is thus given by

$$
\frac{k_{c}^{2}}{k_{\mathrm{TF}}^{2}}=\left[\left(\frac{E_{n p}^{2}}{E_{n n}}-E_{p p}\right) \frac{1}{E_{e e}}+1\right]^{-1}-1,
$$

which reduces to $E_{n n} E_{p p} / E_{n p}^{2}-1$ for $E_{e e}=E_{p p}$. In Fig. 1, we show the calculated values of $k_{c} /(2 \pi / a)$ as a function of density. The predicted values of $k_{c}$ are well below $2 \pi / a$, so our neglect of the $k$ dependence of the parameters is a good first approximation.

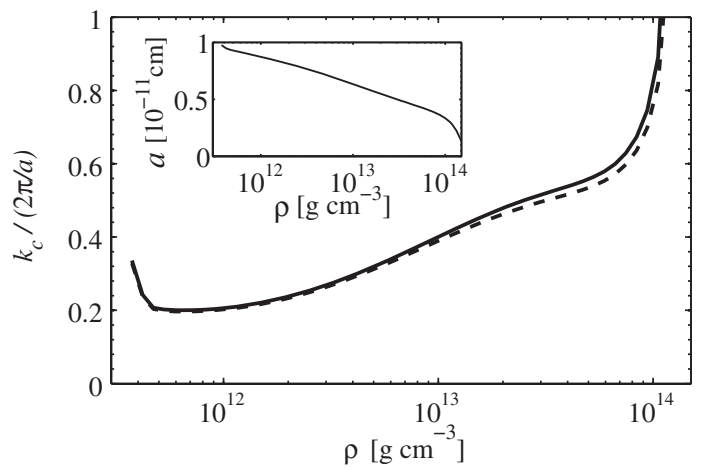

FIG. 1. Wave number for the onset of instability compared with the wave number $2 \pi / a$, which gives the extent of the first Brillouin zone along a cubic axis, as a function of density. The solid line shows results when the shear elastic constants are included [Eq. (7)], while the dashed line shows the results when they are neglected [Eq. (6)]. The inset shows the lattice spacing as a function of density. 
The critical wave number for instability depends very little on whether the original system is a regular solid or a glass, because the main contributions to the energy depend strongly on the volume per nucleus but weakly on the geometry of the cell containing a nucleus. Because $E_{n p}$ is negative, the induced interaction between nuclei favors inhomogeneous matter where regions with a higher density of nuclei also have a higher neutron density.

One may ask whether the instability found above could grow significantly in a neutron star. Using the formalism of Ref. [8], we have estimated the angular frequency and the growth rates of the softest mode. Since the effective neutron-proton interaction $E_{n p}$ is negative, the proton and neutron density variations are in phase for this mode, which corresponds to the one with frequency $k v_{-}$in the notation of that paper. We neglected the shear elastic constant, and the results are shown in Fig. 2 for two densities. As far as the critical wave number is concerned, this calculation is completely equivalent to Eq. (6). For wave numbers different from the critical one, one needs, in addition to the thermodynamic derivatives already considered, the neutron superfluid density, and for our calculations we have assumed that the number density of superfluid neutrons is equal to the density of neutrons outside nuclei. From these results, one sees that the growth times of unstable modes are extremely short on the scale of the lifetime of a neutron star, and this conclusion is unaffected by uncertainties in the neutron superfluid density, which calculations of Chamel indicate could be an order of magnitude smaller than the value we have taken [12].

In the approximations made above, it was assumed that the energy per unit volume depends only on the densities of neutrons, protons, and electrons; thus, effects due to distortion of the crystal from its original cubic form have

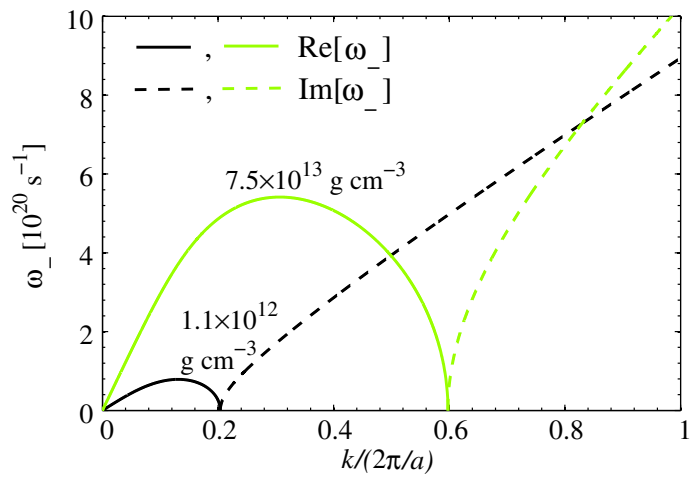

FIG. 2 (color online). Frequency $\omega_{-}=k v_{-}$for the mode in which neutrons and protons move in phase with each other. When $\omega_{-}$is real, its value gives the oscillation frequency (solid lines), while when it is imaginary, the mode is unstable with a growth rate $\operatorname{Im}\left(\omega_{-}\right)$(dashed lines). When the periodicity of the system is properly taken into account, the dispersion relation will have zero slope at the zone boundary $\left(k_{\mathrm{BZ}}=2 \pi / a\right)$ for $\mathbf{k}$ along one of the cubic axes. not been taken into account, and the dispersion relation for modes is independent of the direction of the wave vector. Taking into account the cubic symmetry leads to an anisotropy in the dispersion relation, and we now determine the direction of the wave vector of the most unstable mode for an initial state with a bcc structure, which is the most stable one in the absence of the interaction between nuclei induced by neutrons. For a cubic crystal with no interstitial neutrons, there are three independent elastic constants, which are conveniently taken to be the bulk modulus $B=\left(c_{11}+2 c_{12}\right) / 3$, the modulus $c_{44}$ that describes a shearing perpendicular to one of the cubic axis, and the quantity $c_{11}-c_{12}$, which describes response to a volumeconserving distortion with extension along one cubic axis and compression along one of the other axes. Here we use the standard notation for elastic constants [13]. For bulk stability, $B, c_{11}-c_{12}$, and $c_{44}$ must all be greater than zero. For a bcc Coulomb lattice, the shear elastic constants are dominated by the static lattice contribution, $c_{11}-c_{12} \approx$ $0.10 n_{i} Z^{2} e^{2} / a$, and $c_{44} \approx 0.37 n_{i} Z^{2} e^{2} / a$ [14], where $n_{i}=$ $n_{e} / Z=2 / a^{3}$ is the density of nuclei. For an isotropic solid the ratio $\left(c_{11}-c_{12}\right) / 2 c_{44}$ is unity, while for a Coulomb crystal it is $\sim 0.13$. Crustal material is therefore unusually anisotropic and similar to lithium and plutonium by this measure. For this case, the wave vectors of the most unstable modes associated with a density fluctuation lie along the cubic axes, as Cahn showed in his classic analysis of spinodal instability, in which a binary alloy forms regions with different composition [15]. The phase transition in the neutron star case differs from the usual spinodal instability in that it occurs at a finite wavelength. The instability occurs first when $c_{11}=$ $B+2\left(c_{11}-c_{12}\right) / 3=0$, which is more restrictive than the condition $B=0$ for a crystal with no rigidity to shear. When interstitial neutrons are included, the instability condition is essentially the same, except that the bulk modulus is replaced by the effective proton-proton interaction $E_{p p}(k)-E_{n p}^{2} / E_{n n}$ times $n_{p}^{2}$. The critical wave number is thus given by

$$
E_{p p}\left(k_{c}\right)-\frac{E_{n p}^{2}}{E_{n n}}+\frac{2}{3} \frac{\left(c_{11}-c_{12}\right)}{n_{p}^{2}}=0 .
$$

The solid line in Fig. 1 shows the results of this calculation, and one sees that the inclusion of the shear elastic constants has little effect on the critical wave number. This is because contributions to the shear elastic constants are typically of the order of $Z^{2 / 3} \alpha \sim 0.1$ times the other terms in the instability condition, since the relevant energy scale for the bulk modulus is $n_{e}^{2} \partial \mu_{e} / \partial n_{e}$.

One may ask how robust the instability is to uncertainties in the input nuclear physics. One piece of evidence comes from a simple model for matter at densities not too close to nuclear saturation density: nuclei described by a semiempirical mass formula immersed in a neutron fluid. This 
shows that the induced interaction between nuclei diverges as the density tends to the neutron drip density from above; consequently, the instability appears inevitable near the outer boundary of the inner crust [16]. A second piece of evidence comes from work of Chamel, who calculated thermodynamic derivatives for crustal matter by using an extended Thomas Fermi method and the BSk14 Skyrme interaction, which gives a good fit to observed nuclear masses: he found instability over a broad range of densities [17].

On the basis of the instability analysis alone, it is impossible to predict the equilibrium structure, which depends on higher-order terms in the expansion of the energy in powers of density deviations. However, one would expect the actual structure of the metal to be modulated at wave vectors corresponding to the most unstable modes. In our model, growth rates of modes increase with $k$ for $k>k_{c}$ (Fig. 2), and the maximum growth rate would occur at the zone boundary, which is at a wave vector $(1,0,0) 2 \pi / a$ or some equivalent wave vector, where $a$ is the length of the edge of a cubic unit cell. This corresponds to a doubling of the volume of the unit cell, which becomes a cube with two atoms per unit cell, the one in the middle of the cube being displaced from the center of the cube, as shown in Fig. 3. The transition is thus analogous to that to a ferroelectric in materials such as $\mathrm{BaTiO}_{3}$ [18], but in the neutron star case there is no ferroelectricity, because the displaced nuclei at the center of the cube are identical with the nuclei at the corners. In terrestrial materials under pressure, many examples of incommensurate phases have been discovered [19], and further studies are necessary to determine if such a phase could occur in neutron star crusts.
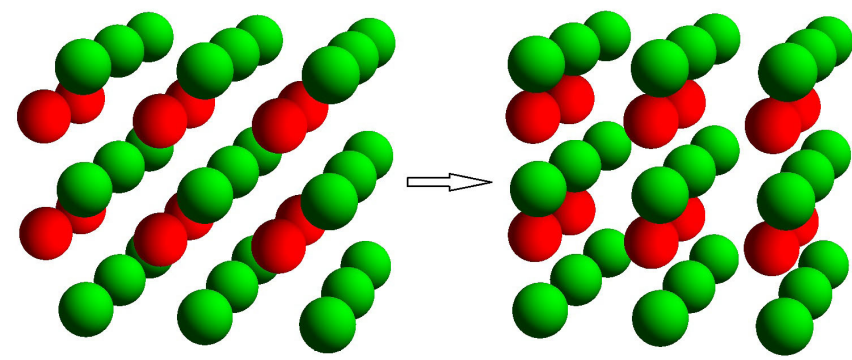

FIG. 3 (color online). Schematic picture of the undistorted bcc lattice (left) and the structure in which the central atoms in the cell are displaced to the right along one of the cubic axes (right), thereby creating a superlattice structure with two atoms per cell. While the nuclei at the centers of cubic cells are identical with those at the corners, for clarity we show nuclei at the centers of unit cells of the undistorted structure as red (dark gray) and those at the corners as green (light gray). If one takes the displacement of the nuclei to lie in the $x$ direction and to be of magnitude $\delta$, one sees that the density of nuclei is higher in the vicinity of the planes $x=\delta / 2-a / 4+\sigma a$ and lower in the vicinity of the planes $x=\delta / 2+a / 4+\sigma a$, where $\sigma$ is an integer.
As regards the basic mechanism, the instability considered here is similar to that previously considered for a uniform fluid of protons, neutrons, and electrons [20-22], which signals the formation of proton clusters (and the high-density boundary of the inner crust) as the density of matter is reduced below nuclear saturation density. In crustal matter, however, the positive charge resides in nuclei, not a homogeneous proton fluid. In both cases, an important element is the reduction of the effective Coulomb interaction at nonzero wave numbers. In the case of the instability of uniform fluids, a stabilizing effect at higher wave numbers is provided by contributions to the energy that depend on density gradients. Identifying the corresponding terms in the case of the instability of a lattice of nuclei is a subject for future work.

In this Letter, we have demonstrated that, because interstitial neutrons in the inner crust behave in a way similar to a second species in a metallic alloy, the physics of the crust is much richer than has been appreciated previously. The phase transition we predict could have consequences for a number of important properties of neutron star crusts and for interpreting observations from space-based instruments. Thermodynamic and transport properties of the crust should be reinvestigated, since they are important in understanding cooling of neutron stars [23], thermal relaxation of the crust [24], and glitch phenomena $[25,26]$. The breaking strain of the crust, which is sensitive to phase transitions of the lattice, is important in a number of contexts. For example, precursor flares prior to the short gamma-ray bursts [27] may be understood as being due to breaking of the crust by tidal forces or as the outcome of a resonance of elastic modes [28]. In Ref. [29], it has been suggested that what the authors refer to as "mountains," nonaxisymmetric distortions of the mass distribution in the inner crust of rapidly rotating, accreting neutron stars, could be detected due to their emission of gravitational waves: the maximum strength of such gravitational wave emission is sensitive to the breaking strain of matter, since this determines the maximum height of a mountain. From experience with terrestrial solids, one knows that the breaking strain of real materials is very different from, and often larger than, that for a perfect lattice, e.g., in martensitic steels.

With the current rapid advances in computational methods in nuclear physics, as well as the increasing amount of experimental data on neutron-rich nuclei that are becoming available, the way is now open to take a fresh look at the properties of matter in the inner crust. In particular, it is important to improve calculations of the thermodynamic derivatives that enter the instability condition. One aspect of the problem that we have not addressed in this Letter is the role of impurity nuclei. In terrestrial materials, these have an enormous influence on physical properties, and their role in neutron stars needs to be reinvestigated. 
We are very grateful to Göran Grimvall for giving us an authoritative introduction to lattice instabilities in metals and to Nicolas Chamel, Pascale Deen, Maxim Mostovoy, David Nelson, and Bertil Sundquist for helpful discussions. C. J. P. thanks Armen Sedrakian for drawing his attention to the possible role of interstitial neutrons in destabilizing matter in the crust of a neutron star. The work of D. K. was supported in part by the J. C. Kempe Memorial Fund and the Rosenfeld Foundation. This work was also supported in part by the Compstar network, COST Action MP1304.

[1] G. Grimvall, B. Magyari-Köpe, V. Ozoliņš, and K. A. Persson, Rev. Mod. Phys. 84, 945 (2012).

[2] See, e.g., P. Haensel, A. Y. Potekhin, and D. G. Yakovlev, Neutron Stars 1. Equation of State and Structure (Springer, New York, 2007), Chap. 2.

[3] The possibility of the lattice in the inner crust of neutron stars being unstable has previously been proposed by A. D. Sedrakian, Astrophys. Space Sci. 236, 267 (1996).

[4] L. D. Landau and E. M. Lifshitz, Statistical Physics (Pergamon, Oxford, 1980), Sec. 96.

[5] Since all protons are in nuclei, the nucleus-nucleus interaction is simply $Z^{2}$ times the proton-proton one.

[6] While the condition for stability must also be satisfied below neutron drip density, the rate at which an instability could develop in such matter would be very long, since it would demand changes in the proton and/or neutron numbers of nuclei, processes which will proceed very slowly when there are no interstitial neutrons. If the $Z$ and $A$ of the nuclei are fixed, the system behaves as a one-component system, and the stability condition is $\partial^{2} E / \partial n^{2}>0$. In the absence of interstitial neutrons, it has been shown that matter consisting of a mixture of two different nuclei, e.g., ${ }^{56} \mathrm{Fe}$ and ${ }^{4} \mathrm{He}$, in an $\mathrm{NaCl}$ structure could have a lower energy than that of the pure phases [F. J. Dyson, Ann. Phys. (N.Y.) 63, 1 (1971)]. Such phases could be of interest in the outer crusts of accreting neutron stars, where locally matter can have more than a single species of nucleus. However, we know of no studies of the stability of such a structure.

[7] J. M. Lattimer and F. D. Swesty, Nucl. Phys. A535, 331 (1991); http://www.astro.sunysb.edu/dswesty/lseos.html.

[8] D. Kobyakov and C. J. Pethick, Phys. Rev. C 87, 055803 (2013).
[9] This conclusion is consistent with earlier work on longwavelength modes [R. I. Epstein, Astrophys. J. 333, 880 (1988); N. Chamel, D. Page, and S. Reddy, Phys. Rev. C 87, 035803 (2013)]. Sedrakian [3] found instability for densities greater than one-third of nuclear density. However, this range of densities, where nuclei are expected to be far from spherical, lies outside the scope of the present Letter, which focuses on lower densities.

[10] D. Pines, Elementary Excitations in Solids (Benjamin, New York, 1963), Chap. 5.

[11] See, e.g., E. L. Pollock and J. P. Hansen, Phys. Rev. A 8, 3110 (1973).

[12] N. Chamel, Phys. Rev. C 85, 035801 (2012).

[13] We follow P. M. Marcus, H. Ma, and S. L. Qiu, J. Phys. Condens. Matter 14, L525 (2002) in using elastic constants defined in terms of derivatives of the Gibbs energy. This maintains the Voigt symmetry and ensures that the elastic constants do not contain contributions proportional to the pressure. With this convention, stability conditions at nonzero pressure have the same form as at zero pressure.

[14] K. Fuchs, Proc. R. Soc. 153, 622 (1936).

[15] J. W. Cahn, Acta Metall. 10, 179 (1962).

[16] D. Kobyakov and C. J. Pethick (to be published).

[17] N. Chamel (private communication); See also N. Chamel, D. Page, and S. Reddy, arXiv:1310.4059.

[18] See, e.g., R. A. Cowley and S. M. Shapiro, J. Phys. Soc. Jpn. 75, 111001 (2006).

[19] M. I. McMahon and R. J. Nelmes, Chem. Soc. Rev. 35, 943 (2006).

[20] H. A. Bethe, G. Baym, and C. J. Pethick, Nucl. Phys. A175, 225 (1971).

[21] C. J. Pethick, D. G. Ravenhall, and C. P. Lorenz, Nucl. Phys. A584, 675 (1995).

[22] K. Hebeler, J. M. Lattimer, C. J. Pethick, and A. Schwenk, Astrophys. J. 773, 11 (2013).

[23] D. G. Yakovlev and C. J. Pethick, Annu. Rev. Astron. Astrophys. 42, 169 (2004).

[24] D. Page and S. Reddy, Phys. Rev. Lett. 111, 241102 (2013).

[25] N. Andersson, K. Glampedakis, W. C. G. Ho, and C. M. Espinoza, Phys. Rev. Lett. 109, 241103 (2012).

[26] N. Chamel, Phys. Rev. Lett. 110, 011101 (2013).

[27] E. Troja, S. Rosswog, and N. Gehrels, Astrophys. J. 723, 1711 (2010).

[28] D. Tsang, J. S. Read, T. Hinderer, A. L. Piro, and R. Bondarescu, Phys. Rev. Lett. 108, 011102 (2012).

[29] C. J. Horowitz and K. Kadau, Phys. Rev. Lett. 102, 191102 (2009). 